\title{
Inhibition of p38mapk Reduces Adipose Tissue Inflammation in Aging Mediated by Arginase-II
}

\author{
Ji Huang ${ }^{a, b} \quad$ Chang Liu $^{a} \quad$ Xiu-Fen Ming ${ }^{a, b} \quad$ Zhihong Yang ${ }^{a, b}$ \\ ${ }^{a}$ Cardiovascular and Aging Research, Department of Endocrinology, Metabolism, and Cardiovascular System, \\ Faculty of Science and Medicine, University of Fribourg, Fribourg, Switzerland; ${ }^{b}$ National Center of Competence in \\ Research "Kidney.CH", Zurich, Switzerland
}

\section{Keywords}

Adipose tissue · Aging · Arginase · Inflammation .

Macrophage $\cdot$ Stromal cells

\section{Abstract}

Background: Adipose tissue inflammation occurs not only in obesity but also in aging and is mechanistically linked with age-associated diseases. Studies show that ablation of the I-arginine-metabolizing enzyme arginase-II (Arg-II) reduces adipose tissue inflammation and improves glucose tolerance in obesity. However, the role of Arg-II in aging adipose tissue inflammation is not clear. Objective: This study investigated the role of Arg-II in age-associated adipose tissue inflammation. Methods: Visceral adipose tissues of young (3-6 months) and old (20-24 months) wild-type (WT) and Arg$\mathrm{II}^{-/-}$mice were investigated. Immunofluorescence confocal microscopy was performed for analysis of macrophage accumulation and cellular localization of arginase and cytokines; expression of arginase and cytokines was analyzed by qRT-PCR or immunoblotting or ELISA; activation of mitogenactivated protein kinases in adipose tissues was analyzed by immunoblotting; and arginase activity was measured by colorimetric determination of urea production. Results: In the old WT mice, there is more macrophage accumulation in the visceral adipose tissues than in Arg-Il knockout animals. An age-associated increase in arginase activity and Arg-II expression in adipose tissues of WT mice is observed. Arg-II knockout enhances Arg-I expression and activity, but inhibits interleukin (IL)- 6 expression and secretion and reduces active p38mapk in aging adipose tissue macrophages and stromal cells. Treatment of aging adipose tissues of WT mice with a specific p38mapk inhibitor SB203580 reduces IL-6 secretion. Conclusions: Arg-II promotes IL-6 production in aging adipose tissues through p38mapk. The results suggest that targeting Arg-II or inhibiting p38mapk could be beneficial in reducing age-associated adipose tissue inflammation.

\section{Introduction}

Aging represents an important challenging health issue of our society [1]. Chronic low-grade inflammation is considered a hallmark for development of many age-associated diseases, including metabolic and cardiovascular diseases [2]. Evidence shows that adipose tissue inflammation is an important contributor not only to the obe-

Ji Huang and Chang Liu equally contributed to this study. 
sity but also to age-associated chronic inflammatory status [3-8]. It has been suggested that adipose tissue is a major source of inflammatory cytokines such as interleukin (IL)- 6 and tumor necrosis factor alpha (TNF- $\alpha$ ) in obesity and aging, termed "inflammaging," contributing to age-associated diseases $[9,10]$. Although many aspects of adipose tissue inflammation are shared by obesity and aging, relatively less work has been done specifically in the context of adipose tissue dysfunction in aging than in obesity [11]. The cellular and molecular mechanisms of adipose tissue inflammation and cytokine production, particularly in physiological aging, are not fully explored.

Studies in recent years demonstrate an important role of the l-arginine-metabolizing enzyme arginase in obesity- and age-associated cardiovascular and metabolic diseases [12-16]. There are 2 isoforms of arginase encoded by separate genes, that is, the cytoplasmic type-I arginase (Arg-I) and mitochondrial type-II arginase (Arg-II) [17]. Both isoforms are involved in 1-arginine metabolism and have shown to share similar functions [18-20], although some controversial findings are described [21]. Both ArgI and Arg-II have been shown to be involved in promoting endothelial nitric oxide synthase 3 dysfunction and adipose tissue inflammation in obesity mouse models [12, 18]. Indeed, mice with endothelial specific Arg-I gene knockout and Arg-II deficiency $\left(\right.$ Arg- $\mathrm{II}^{-/-}$) reveal preserved endothelial function and also decreased adipose tissue inflammation in obesity $[12,18]$. However, the role of Arg-I and Arg-II in age-associated adipose tissue inflammation seems unclear. It is important to note that controversial roles of Arg-I and Arg-II, particularly in regulation of macrophage inflammation and phenotype polarization, are reported. While it is well established that Arg-I is highly associated with the anti-inflammatory macrophage phenotype [21], the role of Arg-II in regulation of macrophage inflammatory response is much less well investigated. Our previous study showed the Arg-II but not Arg-I is markedly enhanced in lipopolysaccharide-stimulated pro-inflammatory macrophages and in macrophages isolated from high-fat-diet-induced obesity, and Arg-II gene deficiency reduces the macrophage inflammatory response in this model $[12,22]$. This finding has been further supported by studies from other groups $[23,24]$. However, the anti-inflammatory function of ArgII in macrophages is also described in the literature [2527]. To our knowledge, the role of Arg-II in age-associated adipose tissue macrophage inflammation and the underlying molecular mechanisms are not clear. Moreover, adipose tissue contains many other cell types which are also involved in adipose tissue inflammation [11].
Mitogen-activated protein kinases (MAPKs), including extracellular signal-regulated kinase (ERK), c-Jun amino-terminal kinase (JNK), and p38mapk, are importantly involved in the regulation of inflammation processes in obesity $[28,29]$. It has been reported that p38mapk plays a role in pro-inflammatory cytokine biosynthesis (e.g., TNF- $\alpha$, IL-1 $\beta$, and IL-6) [30, 31]. Adipose tissue-specific deficiency in or pharmacological inhibition of $\mathrm{p} 38 \mathrm{mapk}$ shows protection of mice against obesity [32]. Since aging is often accompanied by obesity which interferes with interpretation of organ functional changes during aging $[19,33]$, we investigated the role of Arg-II and p38mapk in age-associated adipose tissue inflammation in a mouse model. In the present study, we demonstrate that p38mapk is the signal transduction pathway that mediates Arg-II-induced adipose tissue inflammation, particularly IL-6 production in aging adipose tissues.

\section{Materials and Methods}

\section{Materials}

All chemicals including those used for immunoblotting and antibodies (Abs) against $\beta$-actin (A5441) and tubulin (T5168) were obtained from Sigma (Buchs, Switzerland), and RPMI-1640 was purchased from Amimed (Muttenz, Switzerland). The p38mapk inhibitor SB203580 (4-[4-fluorophenyl]-2-[4methylsulfinylphenyl]-5-[4-pyridyl] $1 H$-imidazole) was purchased from Calbiochem (Lucerne, Switzerland). Rat Ab against Mac-2 (eBioM3/38, 14-5301-82) was from ebioscience ${ }^{\mathrm{TM}}$. Rabbit Abs against Arg-I (\#93668) and Arg-II (\#55003), phosphop38mapk (Thr180/Tyr182, \#4511), IL-6 (\#12912), phospho-ERK (Thr202/Tyr204, \#4377), phospho-stress-activated protein kinases/JNK (Thr183/Tyr185, \#9251), and stress-activated protein kinases/JNK (\#9252) were from Cell Signaling Technology (Allschwil, Switzerland). Mouse Ab against p38mapk (\#612168) and mouse $\mathrm{Ab}$ against pan-ERK (\#610123) were from BD Biosciences (NJ, USA). Mouse Ab against TNF- $a$ (ab1793) was from Abcam (Cambridge, UK). Bio-Rad DC ${ }^{\mathrm{TM}}$ protein assay kit was from Bio-Rad Laboratories (Basal, Switzerland). Alexa Fluor 680-conjugated anti-mouse IgG (A21057) was from Molecular Probes Invitrogen (Lucerne, Switzerland). IRDye800-conjugated anti-rabbit IgG (\#926-32211) was from LI-COR Biosciences (Bad Homburg, Germany). Alexa Fluor 488-conjugated goat anti-rabbit IgG $(\mathrm{H}+$ L) secondary Ab (A-11008) and Alexa Fluor 546-conjugated goat anti-rat IgG $(\mathrm{H}+\mathrm{L})$ secondary Ab (A-11081) were from Thermo Fisher Scientific.

\section{Animals}

The Arg-II-deficient mice (Arg- $\mathrm{II}^{-/-}$) were kindly provided by Dr. William O'Brien [34] and backcrossed to C57BL/6J for more than 8 generations. Genotyping was performed by PCR as previously described [34]. The wild-type (WT) and Arg-II ${ }^{-/-}$offspring from heterohetero cross were interbred to obtain WT and Arg$\mathrm{II}^{-/-}$mice, respectively, for experiments. Both young (3-6 months 
old) and old (20-24 months) female WT and Arg $\mathrm{II}^{-/-}$mice were maintained on a 12-h light-dark cycle and fed standard chow diet and tap water according to the local guidelines for animal experimentation. Since Arg-II expression in various organs is significantly higher in females than in males and female animals exhibit much stronger aging phenotype than males $[35,36]$, we decided to analyze age-associated adipose tissue inflammation in female animals. Peri-gonadal fat tissue is the most widely used visceral fat tissue for analyzing adipose tissue function; therefore, peri-uterus fat tissue was collected for the study. In some cases, mesenteric fat tissues were also included. The animals were anesthetized with $\mathrm{xy}$ lazine $(10 \mathrm{mg} / \mathrm{kg}$ body weight intraperitoneally) and ketamine (100 $\mathrm{mg} \mathrm{kg}^{-1}$ body weight intraperitoneally) and euthanized. Periuterus fat was collected for preparation of conditional medium and paraffin sections for immunostaining or snap frozen directly in liquid nitrogen and kept at $-80^{\circ} \mathrm{C}$ until further biochemical analyses.

\section{Immunofluorescence Staining and Confocal Microscope Imaging}

For co-immunofluorescence staining of Mac-2, Arg-I, Arg-II, and p-p38mapk, peri-uterus fat from WT and Arg- $\mathrm{II}^{-l-}$ mice was isolated, fixed with $3.7 \%$ paraformaldehyde, and embedded in paraffin. After deparaffinization in xylene, hydration in ethanol, and antigen retrieval either in citrate-EDTA buffer $(10 \mathrm{mmol} / \mathrm{L}$ citric acid, $2 \mathrm{mmol} / \mathrm{L}$ EDTA, and $0.05 \%$ Tween 20, pH 6.2) for Mac-2, Arg-I, and p-p38mapk or in EDTA buffer $(1 \mathrm{mmol} / \mathrm{L}$ EDTA, $0.05 \%$ Tween $20, \mathrm{pH} 8.0$ ) for Arg-II at $95-100^{\circ} \mathrm{C}$ for $20 \mathrm{~min}$, paraffin-embedded sections $(5 \mu \mathrm{m})$ were blocked with $1 \%$ bovine serum albumin (BSA) and 10\% goat serum in PBS for $1 \mathrm{~h}$, and then incubated with primary Abs (Mac-2 1:200, Arg-I 1:100, Arg-II 1:300, and p-p38mapk 1:50) at $4^{\circ} \mathrm{C}$ overnight. Subsequently, sections were incubated with fluorescence-labeled secondary Abs at room temperature for $2 \mathrm{~h}$, followed by counterstaining with 300 nmol/L 4'6-diamidino-2-phenyl-indole, dihydrochloride (Invitrogen) for $3 \mathrm{~min}$. For co-immunofluorescence staining of Mac-2 and IL-6, peri-uterus fat was isolated and fixed with 3.7\% paraformaldehyde. Once fixed, fat tissues were transferred to $30 \%$ sucrose/PBS at $4^{\circ} \mathrm{C}$ overnight and then embedded in Tissue-Tek optimum cutting temperature compound (SAK-4583; Sakura Finetek) and frozen in liquid nitrogen. Frozen sections $(20 \mu \mathrm{m})$ were cut with a cryostat (388159; Thermo Scientific HM525 NX). Sections were mounted on Fisher Superfrost Plus slides and stored at $-20^{\circ} \mathrm{C}$ until immunostaining. After $10 \mathrm{~min}$ of rehydration in PBS, sections were incubated with blocking buffer for $1 \mathrm{~h}$, followed by incubation with primary Abs and secondary Abs, respectively. Then, the sections were counterstained with $300 \mathrm{nmol} / \mathrm{L} 4^{\prime} 6$-diamidino-2-phenyl-indole, dihydrochloride for $3 \mathrm{~min}$. Negative control for staining was performed by omitting the primary $\mathrm{Ab}$ (using blocking buffer alone). Immunofluorescence signals were visualized under a Leica DIM6000 confocal microscope. The number of macrophage accumulation was determined by counting the number of Mac-2-positive cells and expressed as cells per field.

\section{Real-Time qRT-PCR Analysis}

Two-step qRT-PCR analysis was performed to evaluate mRNA expression of the endogenous Arg-I, Arg-II, and inflammatory cytokines in mouse fat tissues as described previously [18]. Total RNA was extracted from fat tissues with Trizol reagent (Molecular Research Center, Inc., Cincinnati, OH, USA) following the sup- plier's protocol. mRNA expression levels of all genes were normalized to the reference gene glyceraldehyde 3-phosphate dehydrogenase (GAPDH). The primer sequences of mouse origin $(\mathrm{m})$ are as follows:

mArg-I-F: GGAATCTGCATGGGCAACCTGTGT;

mArg-I-R: AGGGTCTACGTCTCGCAAGCCA.

mArg-II-F: CCCCTTTCTC TCGGGGACAGAA;

mArg-II-R: GAAAGGAAAGTGGCTGTCCA.

IL6-F: GACAACCACGGCCTTCCCTA;

IL6-R: GCCTCCGACTTGTGAAGTGGT.

IL-1 $\beta$-F: GCAACTGTTCCTGAACTCAACT;

IL-1 $\beta$-R: TCTTTTGGGGTCCGTCAACT.

iNOS-F: GGCAAACCCAAGGTCTACGTT;

iNOS-R: TCGCTCAAGTTCAGCTTGGT.

MCP1-F: AGCACCAGCCAACTCTCAC;

MCP1-R: TCTGGACCCATTCCTTCTTG.

mKC-F: CAATGAGCTGCGCTGTCAGTG;

mKC-R: CTTGGGGACACCTTTTAGCATC.

mTNF- $a-F:$ GGCAGGTCTACTTTGGAGTCATTGC;

mTNF- $\alpha-R:$ ACATTCGAGGCTCCAGTGAATTCGG.

mGAPDH-F: ACCCAGAAGACTGTGGATGG;

mGAPDH-R: ACACATTGGGGGTAGGAACA.

\section{ELISA}

The protein level of IL- 6 in the conditioned medium of DMEM containing $0.2 \%$ BSA was evaluated by using the ELISA MAX Deluxe from BioLegend, according to the manufacturer's instructions (Lucerna Chem AG, Luzern, Switzerland).

\section{Immunoblotting Analysis}

Tissue lysate preparation, sodium dodecyl sulfate-polyacrylamide gel electrophoresis, and transfer of protein from within the sodium dodecyl sulfate gels to Immobilon-P membranes (Millipore) were performed as previously described [18]. The resultant membrane was incubated overnight with the corresponding first antibody at $4^{\circ} \mathrm{C}$ with gentle agitation after blocking with $5 \%$ skimmed milk. The protein was then decorated with corresponding anti-mouse (Alexa Fluor 680-conjugated) or anti-rabbit (IRDye 800-conjugated) secondary antibodies. Signals were visualized with Odyssey Infrared Imaging System (LI-COR Biosciences). Quantification of the signals was performed in NIH Image 1.62 software.

\section{Arginase Assay}

Arginase activity in adipose tissue lysates was measured by colorimetric determination of urea formed from 1 -arginine in an in vitro activity assay, as previously described [18]. Briefly, fat powders were lysed in lysis buffer containing $10 \mathrm{mmol} / \mathrm{L}$ Tris- $\mathrm{HCl}$ (pH 7.4), $0.4 \%$ Triton X-100, $10 \mu \mathrm{g} / \mathrm{mL}$ leupeptin, and $0.1 \mathrm{mmol} / \mathrm{L}$ phenylmethylsulfonyl fluoride. Samples were centrifuged at 13,000 $\mathrm{rpm}$ at $4^{\circ} \mathrm{C}$ for $10 \mathrm{~min}$, and protein concentration of the supernatant was determined by the Lowry method (Bio-Rad). For measurement of arginase activity, an equal amount of the lysates was added to $50 \mu \mathrm{L}$ of Tris- $\mathrm{HCl}(10 \mathrm{mmol} / \mathrm{L}$ [pH 7.4]) containing 5 $\mathrm{mmol} / \mathrm{L} \mathrm{MnCl}_{2}$. Arginase was then activated by heating the mixture at $56^{\circ} \mathrm{C}$ for $10 \mathrm{~min}$. The hydrolysis reaction of l-arginine by arginase was conducted by incubating the mixture containing activated arginase with $100 \mu \mathrm{L}$ of l-arginine (100 mmol/L [pH 9.6]) at $37^{\circ} \mathrm{C}$ for $1 \mathrm{~h}$. For colorimetric determination of urea, $1 \mathrm{~mL}$ of chromogenic reagent consisting of 1 volume of 3\% 2,3-butanedi- 

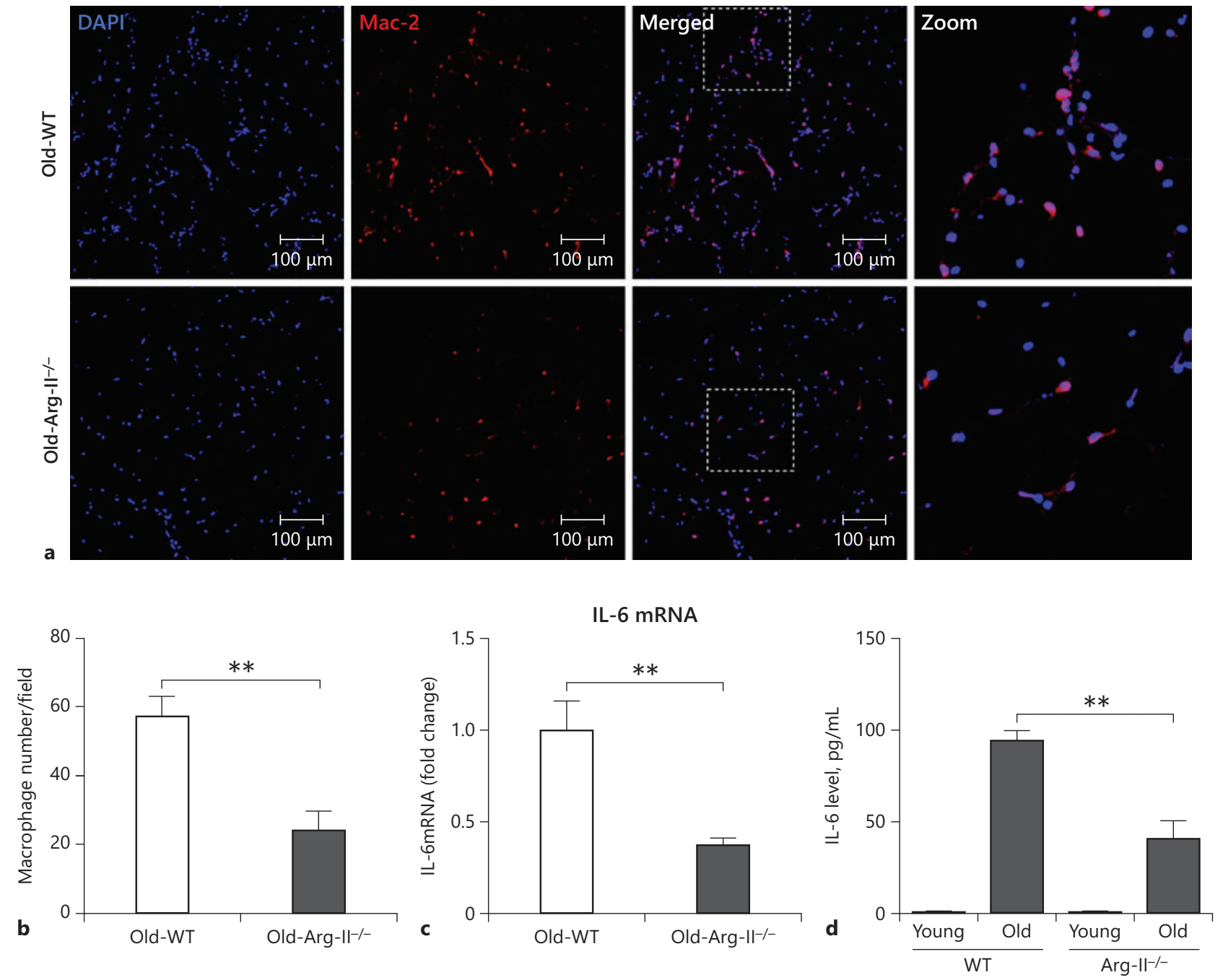

Fig. 1. Decreased macrophage accumulation in the adipose tissue of old Arg-II deficient (Arg- $\mathrm{II}^{-/-}$) mice. a Representative confocal images of macrophage accumulation in the peri-uterus fat tissue of old WT and Arg-II ${ }^{-/-}$mice as assessed by immunofluorescence staining of paraffin-embedded sections with antibody against the macrophage marker Mac-2 (red), followed by counterstaining of the nuclei with DAPI (blue). Scale bar $=100 \mu \mathrm{m}$. b Quantification of the macrophage number per field is shown in the graph below

one monoxime and 29 volumes of the acid solution mixture $\left(\mathrm{H}_{2} \mathrm{SO}_{4}: \mathrm{H}_{3} \mathrm{PO}_{4}: \mathrm{H}_{2} \mathrm{O}\right.$ 1:3:7) was added, and the mixture was then heated at $100^{\circ} \mathrm{C}$ for $30 \mathrm{~min}$. After placing the samples in the dark for $10 \mathrm{~min}$ at room temperature, the urea concentration $(\mu \mathrm{mol} / \mathrm{L})$ was determined spectrophotometrically by the absorbance at 492 $\mathrm{nm}$. The amount of urea produced ( $\mu \mathrm{mol} / \mathrm{L}$ urea/ $\mu \mathrm{g}$ protein) was used as an index for arginase activity. (left panel), $n=4$. c qRT-PCR analysis of IL- 6 mRNA expression in the peri-uterus fat tissue, $n=8$. $\mathbf{d}$ IL- 6 protein level in the conditioned medium from peri-uterus fat tissues of mice measured by ELISA, $n=4$. $n$ indicates the number of animals of each experimental group. ${ }^{* *} p<0.01$ between the indicated groups. Arg, arginase; WT, wild type; DAPI, 4'6-diamidino-2-phenyl-indole, dihydrochloride.

\section{Preparation of Adipose Tissue-Conditioned Medium from} Mouse

The animals were anesthetized with xylazine $(10 \mathrm{mg} / \mathrm{kg}$ body weight intraperitoneally) and ketamine (100 $\mathrm{mg} \mathrm{kg}^{-1}$ body weight intraperitoneally) and euthanized; $800 \mathrm{mg}$ of peri-uterus fat were cut into small pieces and incubated in $1 \mathrm{~mL}$ of DMEM supplemented with $0.2 \% \mathrm{BSA}$ in the absence or presence of the p38mapk inhibitor SB203580 $(10 \mu \mathrm{mol} / \mathrm{L})$ at $37^{\circ} \mathrm{C}$ for $24 \mathrm{~h}$. The conditioned medium was then collected and kept at $-80^{\circ} \mathrm{C}$ till further analyses. 


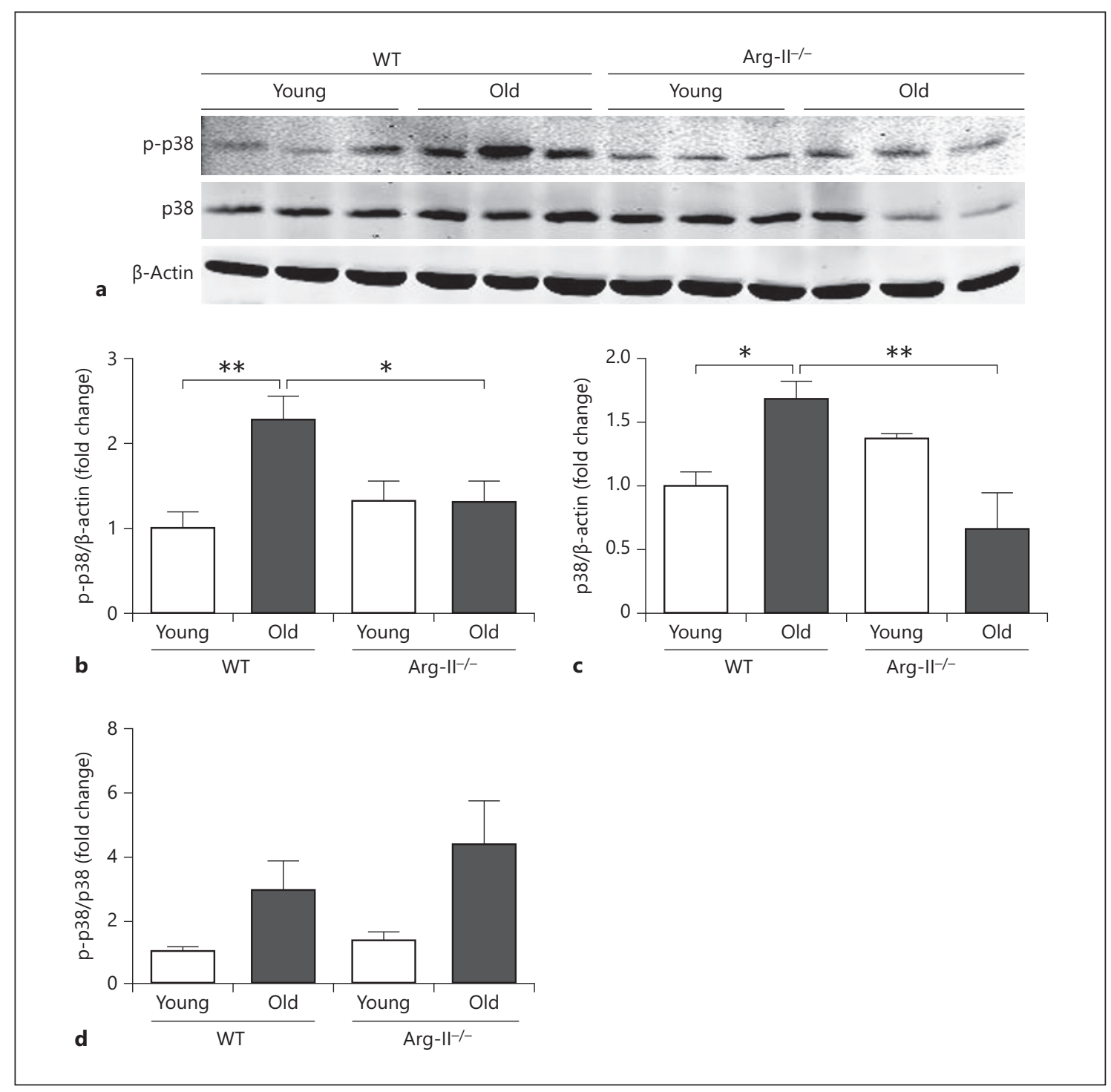

Fig. 2. Arg-II deficiency prevents age-associated increase in active p38mapk in adipose tissues. a Peri-uterus fat tissue protein lysates were prepared and subjected to immunoblotting analysis of phosphor-p38 (p38-p) and total p38 (p38-t). $\beta$-Actin was used as loading control. b-d Quantification of the signals shown in (a): $\mathrm{p} 38$-p/ $\beta$-actin (b), total p38/ $\beta$-actin (c), p38-p/total p38 (d). $n=6 . n$ indicates the number of animals of each experimental group. ${ }^{*} p<0.05,{ }^{* *} p<0.01$ between the indicated groups. Arg, arginase; WT, wild type.

\section{Statistics}

Data are given as mean \pm SEM. In all experiments, $n$ represents the number of experiments or animals. Statistical analysis was performed with unpaired $t$ test or ANOVA with Bonferroni posttest adjustment. Differences in mean values were considered significant at $p<0.05$.

\section{Results}

\section{Arg-II Deficiency Reduces Adipose Tissue}

Inflammation in Aging

In old WT mice, adipose tissue macrophage accumulation as demonstrated by Mac-2-positive cells forming crown-like structures was significantly reduced in the age-matched Arg-II ${ }^{-/-}$mice (Fig. 1a, b). The age-associated adipose tissue macrophage accumulation was cor- 


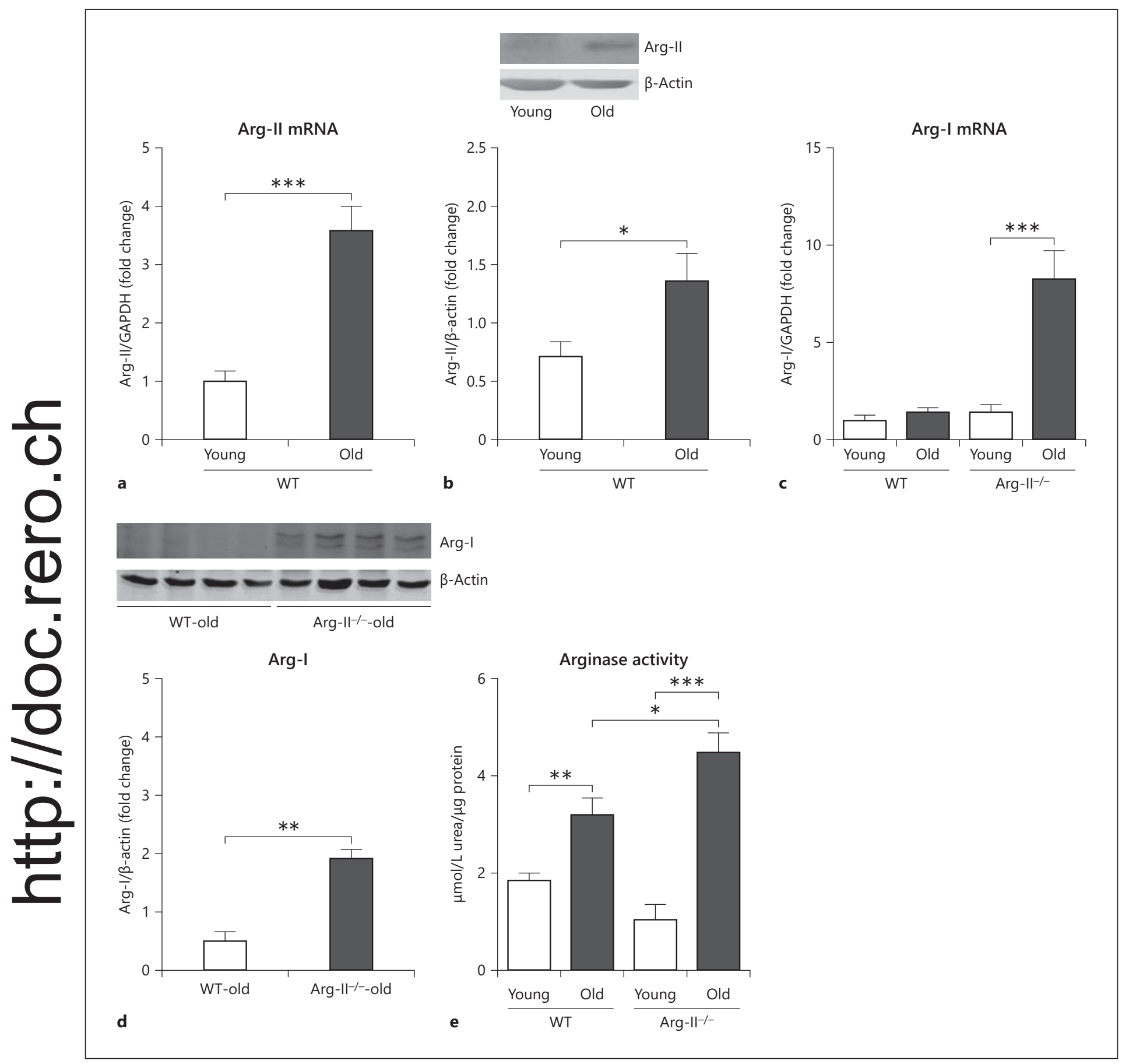

Fig. 3. Arg-II expression and arginase activity are enhanced in adipose tissues in old WT mice. Protein and RNA extracts of periuterus fat tissues or mesenteric fat tissues were prepared from young (3-6 months old) and old (20-24 months old) female mice of both genotype and subjected to qRT-PCR analysis, $n=6(\mathrm{a})$, and immunoblotting of Arg-II, $n=5$ (b); qRT-PCR analysis of Arg-I in young and old mice of both genotypes, $n=6$ (c); immunoblotting analysis of Arg-I, $n=4(\mathbf{d})$; arginase activity assay in young and old mice of both genotypes, $n=6(\mathrm{e}) . n$ indicates the number of animals of each experimental group. ${ }^{*} p<0.05,{ }^{* *} p<0.01$, ${ }^{* * *} p<0.001$ between the indicated groups. Arg, arginase; WT, wild type; GAPDH, glyceraldehyde 3-phosphate dehydrogenase. 

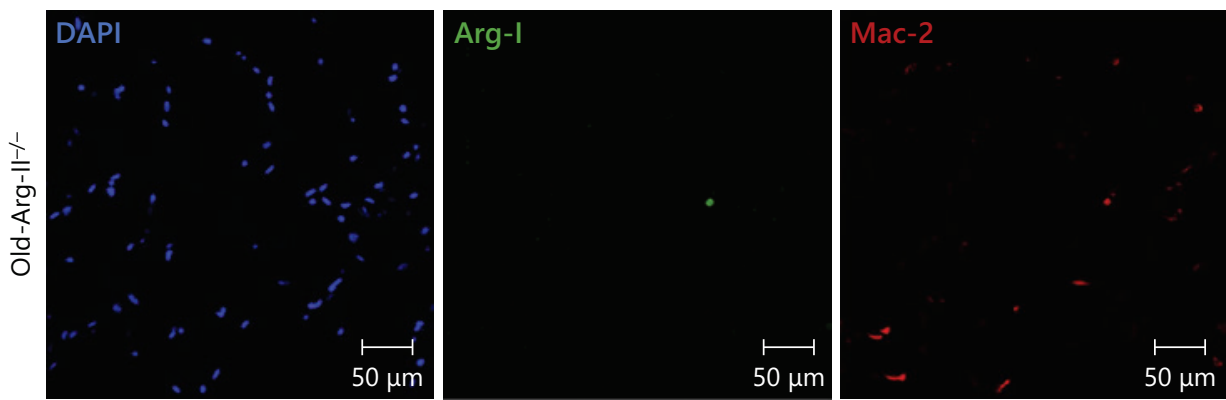

Merged
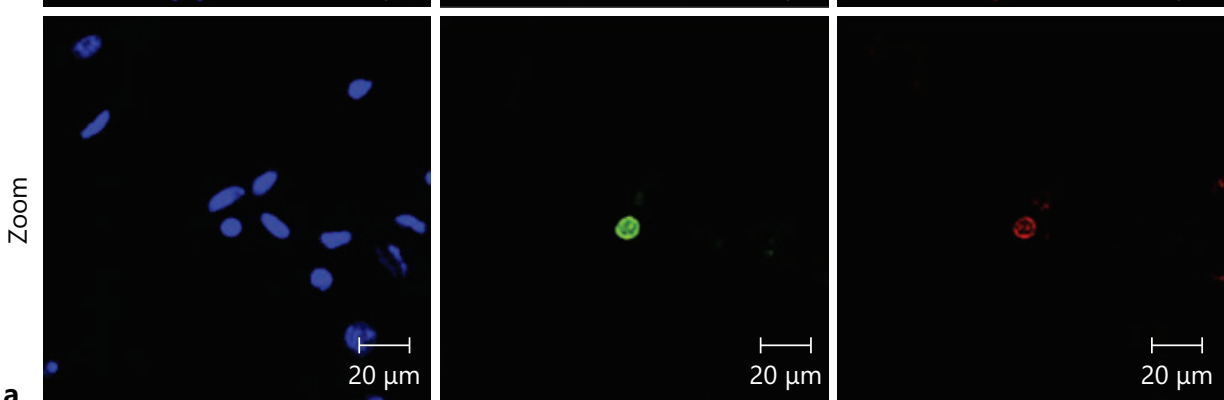

$50 \mu \mathrm{m}$
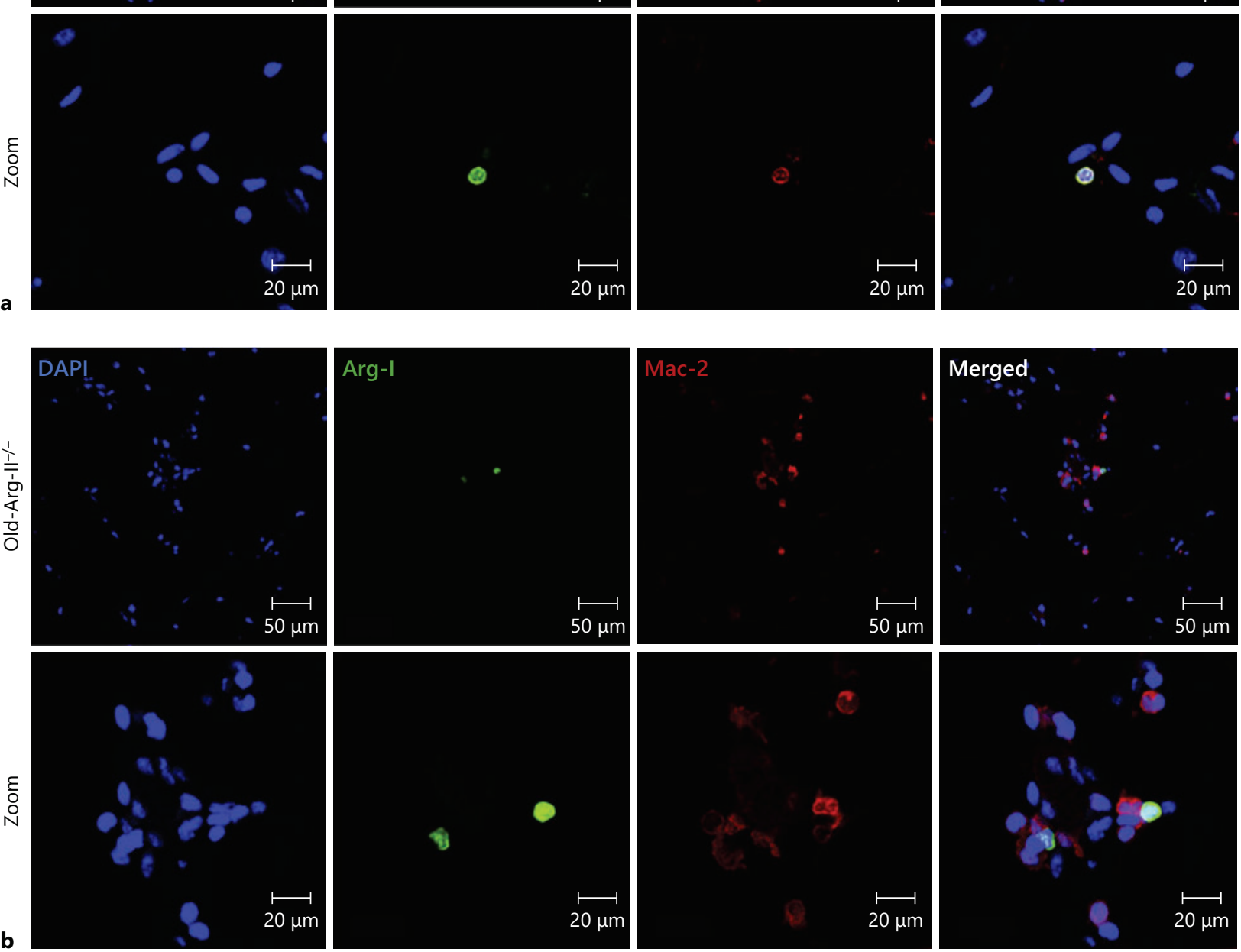

Fig. 4. Localization of Arg-I in adipose tissues of old-Arg-II ${ }^{-/}$ mice. Representative confocal images of the localization of Arg-I in adipose tissues of old-Arg- $\mathrm{II}^{-/-}$mice as assessed by immunofluorescence staining of paraffin-embedded sections from periuterus fat tissues of old Arg- $\mathrm{II}^{-/-}$mice with antibodies against ArgI (green) and Mac-2 (red), followed by counterstaining of the nuclei with DAPI (blue). Scale bar $=50 \mu \mathrm{m}$. Arg-I is expressed in

Mac-2-positive cells (ATMs) (a); Arg-I is also expressed in Mac2-negative cells (ATSCs) in peri-uterus fat tissues (b). Shown are representative images from 3 independent series of experiments. Arg, arginase; WT, wild type; DAPI, 4'6-diamidino-2-phenyl-indole, dihydrochloride; ATMs, adipose tissue macrophages; ATSCs, adipose tissue stromal cells. 
related with IL-6 mRNA expression and protein production, which was significantly reduced in the Arg- $\mathrm{II}^{-/-}$ mice (Fig. 1c, d). A trend of decrease in mRNA levels of IL-1 $\beta$ and murine keratinocyte-derived chemokine (mKC, the murine orthologue of human IL-8) in Arg$\mathrm{II}^{-/-}$mouse adipose tissues was observed (see online suppl.Fig. 1a,b; seewww.karger.com/doi/10.1159/000507635 for all online suppl. material). No difference in mRNA levels of monocyte chemoattractant protein 1 and inducible nitric oxide synthase could be detected between old $\mathrm{WT}$ and age-matched Arg- $\mathrm{II}^{-/-}$mice (online suppl. Fig. $1 c, d)$. Also TNF- $\alpha$ expression either at mRNA or protein levels was not different between the 2 groups (online suppl. Fig. 1e, f). Therefore, the following experiments were focusing on adipose tissue IL-6.

\section{Arg-II Deficiency Reduces Total p38mapk Protein Level in Aging Adipose Tissues}

In accordance with the enhanced adipose tissue inflammation with aging, an increase in levels of active p38mapk (p-p38mapk) in adipose tissues of the old WT mice was observed, which was prevented by Arg-II gene deficiency (Fig. 2a, b). The reduced p-p38mapk in Arg$\mathrm{II}^{-/-}$adipose tissues is attributed to the decreased total p38mapk protein level (Fig. 2a, c), but not to an enhanced activation of $\mathrm{p} 38 \mathrm{mapk}$ as reflected by the comparable $\mathrm{p}$ p38mapk/total p38mapk ratio (Fig. 2d). No significant differences in activation of ERK1/2 and JNK pathways were observed between old WT and Arg-II ${ }^{-/-}$adipose tissues (online suppl. Fig. 2).

\section{Enhanced Arg-I Expression/Activity in Old Arg-II-/- Adipose Tissues}

There was a significantly increase in Arg-II mRNA expression in the adipose tissue of old WT mice (Fig. 3a); the absolute gene expression level was low as reflected by the high CT value in qRT-PCR (data not shown). Although immunoblotting was not sensitive enough to detect Arg-II protein level in the peri-gonadal fat, it showed a significant increase in mesenteric fat in old mice (Fig. 3b). In aging adipose tissues, there was no increase in Arg-I mRNA expression in WT mice (Fig. 3c). However, Arg-I mRNA and protein levels were significantly augmented in the old Arg-
$\mathrm{II}^{-/-}$mice (Fig. 3c, d), which shall account for the enhanced arginase activity in the old Arg-II ${ }^{-/-}$animals (Fig. 3e).

Cellular Localization of Arginase, Active p38mapk, and IL-6 in Adipose Tissue Cells in Aging

We next investigated cellular localization of arginase, active $\mathrm{p}-\mathrm{p} 38 \mathrm{mapk}$, and inflammation in aging adipose tissues. Immunofluorescence confocal microscopy demonstrated that Arg-I was expressed in both Mac-2 (macrophage marker)-positive (Fig. 4a) and Mac-2-negative cells (Fig. 4b) in the adipose tissue of old Arg- $\mathrm{II}^{-/-}$mice, suggesting that Arg-I was expressed in both adipose tissue macrophages (ATMs) and adipose tissue stromal cells (ATSCs). Arg-II was detected not only in the Mac2-positive macrophages (Fig. 5a, upper and middle panels) of old WT mice but also in Mac2-negative cells (Fig. 5a, lower panel). As expected, Arg-II protein was absent in the old Arg-II ${ }^{-/-}$mice (Fig. 5a). Since a more pronounced active p-p38mapk was found with aging in WT mice, cellular localization of $\mathrm{p}$-p38mapk was also investigated in old mouse adipose tissues. The results show that $\mathrm{p}$-p38mapk was mainly found in Mac-2-positive ATMs and could also be detected in some Mac-2-negative cells, that is, ATSCs (Fig. 6). In line with the results shown in Figure 1a, there were much less macrophages in the adipose tissue of old Arg-II ${ }^{-/-}$mice than the WT mice (Fig. 6). Moreover, IL-6 was also found in Mac-2-positive ATMs and Mac2-negative ATSCs of old WT mice, which was reduced in Arg$\mathrm{II}^{-/-}$mice (Fig. 7a), suggesting that Arg-II deficiency reduces IL-6 expression in aging adipose tissues.

\section{p38mapk Is Required for Age-Associated IL-6 Release from Adipose Tissues}

To assess the role of p38mapk in Arg-II-promoted IL-6 production in aging adipose tissues, adipose tissues from old WT mice were incubated in cell culture medium in the absence or presence of the specific p38mapk inhibitor SB203580 for $24 \mathrm{~h}$ prior to the collection of the conditioned medium. As shown in Figure 7b, SB203580 significantly reduced IL-6 protein release from the adipose tissues of old WT mice, demonstrating that 338 mapk is required for age-associated, Arg-II-promoted IL-6 release from adipose tissues.
Fig. 5. Localization of Arg-II in adipose tissues in aging. Confocal images of the localization of Arg-II in adipose tissues of old WT mice (a) and Arg- $\mathrm{II}^{-/-}$mice (b) as assessed by immunofluorescence staining of paraffin-embedded sections with antibodies against Mac-2 (red) and Arg-II (green), followed by counterstain- ing of the nuclei with DAPI (blue). Scale bar $=100 \mu \mathrm{m}$ for lower amplification in the upper panel of $\mathbf{a}, \mathbf{b}$ or $50 \mu \mathrm{m}$ for zoomed images. Shown are representative images from 3 independent series of experiments. Arg, arginase; WT, wild type; DAPI, 4'6-diamidino-2-phenyl-indole, dihydrochloride. 


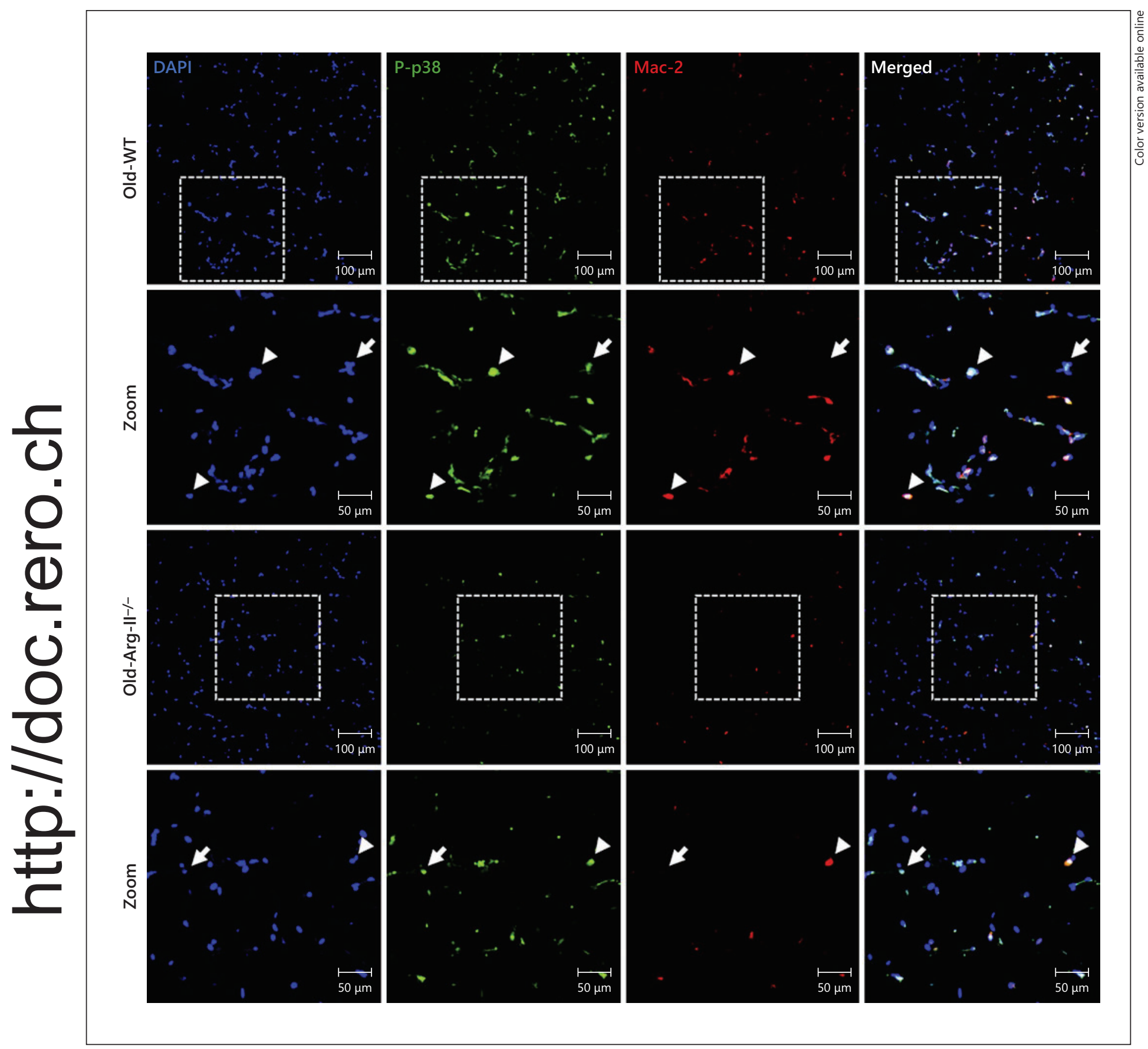

Fig. 6. Localization of active p-p38mapk in adipose tissues in aging. Confocal images of the localization of active p-p38mapk in adipose tissues of old mice as assessed by immunofluorescence staining of paraffin-embedded sections from peri-uterus fat tissues with antibodies against p-p38 (green) and Mac-2 (red), followed by counterstaining of the nuclei with DAPI (blue). Scale bar $=100$

$\mu \mathrm{m} . \mathrm{p}-\mathrm{p} 38$ is mainly expressed in Mac-2-positive cells (ATMs, arrowhead), and also in Mac-2-negative cells (ATSCs, arrow) in peri-uterus fat tissues. Shown are representative images from 3 independent series of experiments. Arg, arginase; WT, wild type; DAPI, 4'6-diamidino-2-phenyl-indole, dihydrochloride; ATMs, adipose tissue macrophages; ATSCs, adipose tissue stromal cells. 

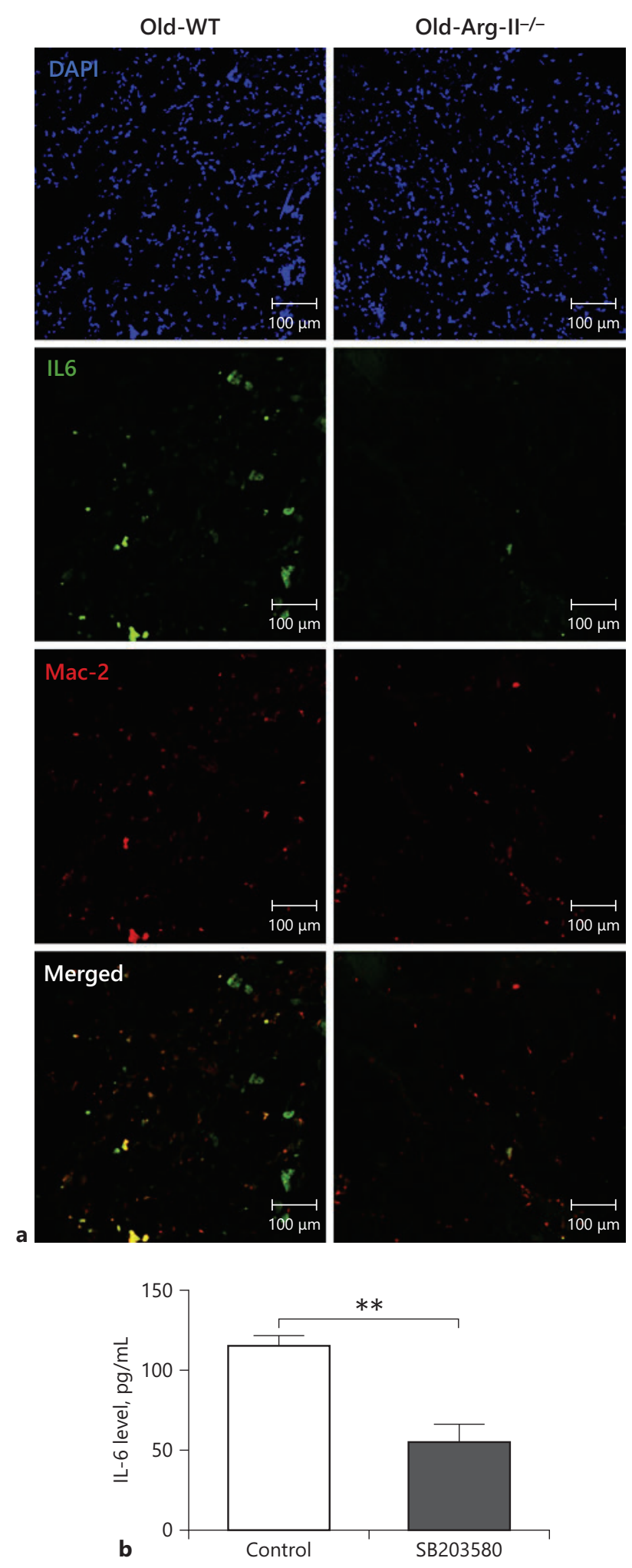

\section{Discussion}

In the present study, we confirmed previous findings that aging is indeed associated with chronic adipose tissue inflammation [37]. Furthermore, the age-associated adipose tissue inflammation is inhibited in Arg- $\mathrm{II}^{-/-}$mice. Previous studies demonstrated an upregulation of Arg-II expression in aging endothelial cells, promoting cellular senescence and dysfunction [12]. Arg-II is also upregulated in macrophages and promotes pro-inflammatory cytokine release, including IL- 6 and TNF- $\alpha$, contributing to glucose intolerance in a high-fat-diet-induced obesity mouse model [18]. In line with this finding, mice with Arg-II deficiency $\left(\mathrm{Arg}-\mathrm{II}^{-/-}\right)$are protected from vascular functions, and metabolic disorders and adipose tissue inflammation in obesity $[18,24]$. Since aging is often accompanied by obesity which interferes with interpretation of organ functional changes during aging [19], the present study investigated the role of Arg-II in adipose tissue inflammation of a physiological aging mouse model [36]. In this aging model, we showed previously that Arg-II is upregulated in pancreatic acinar cells and promotes $\beta$-cell dysfunction via paracrine release of TNF- $\alpha$, contributing to age-associated glucose intolerance [36]. However, there is no difference in TNF- $\alpha$ mRNA and protein levels found in visceral adipose tissues from these old WT and Arg- $\mathrm{II}^{-/-}$mice, suggesting a tissue specificity of age-associated inflammation in aging.

It has been reported that both Arg-I and Arg-II are upregulated in vascular endothelial cells and perivascular adipose tissues in obesity models $[19,24]$. Similar to the obesity models, our present aging model shows an enhanced arginase activity, which is accompanied by an increase in Arg-II mRNA expression in visceral adipose tissues in aging. Arg-I mRNA in the aging adipose tissue is, however, increased only under the condition of Arg-II

Fig. 7. Origin of IL-6 and p38mapk-requirement for IL-6 production in adipose tissues in aging. a Confocal images of the localization of IL- 6 in adipose tissues of old mice as assessed by immunofluorescence staining of cryosections from peri-uterus fat tissues with antibodies against IL-6 (green) and Mac-2 (red), followed by counterstaining of the nuclei with DAPI (blue). Scale bar $=100 \mu \mathrm{m}$. Shown are representative images from 3 independent series of experiments. b Ex vivo effects of the p38mapk inhibitor SB203580 $(10 \mu \mathrm{mol} / \mathrm{L}, 24 \mathrm{~h})$ on IL- 6 production/release from peri-uterus fat tissues of old WT mice measured by ELISA. Data shown are mean \pm SEM from 3 individual animals of each experimental group. ${ }^{* *} p<0.01$ between the indicated groups. IL, interleukin; Arg, arginase; WT, wild type; DAPI, 4'6-diamidino-2-phenyl-indole, dihydrochloride; SEM, standard error of the mean. 
deficiency. The results implicate a compensatory increase in Arg-I in the adipose tissue of Arg-II ${ }^{-/-}$mice, a similar finding reported in gastric tissues [27]. The results also suggest a different regulation of arginase isoforms in adipose tissues in obesity and aging. Interestingly, the enhanced Arg-I levels in the aging adipose tissue under ArgII deficiency are correlated with decreased age-associated inflammation, that is, macrophage accumulation and IL-6 levels in the adipose tissue, which could be due to the fact that Arg-I is upregulated in the M2-like macrophages which play an anti-inflammatory role [38-42]. In contrast to Arg-I, Arg-II is often associated with pro-inflammatory M1-like phenotype cells [18, 41], although controversies are reported in the literature [18, 22-27]. The reasons for the controversies are not clear; it is most likely due to the different inflammation disease models. Nevertheless, our present study shows decreased macrophage accumulation and IL- 6 expression in the aging adipose tissue of Arg- $\mathrm{II}^{-/-}$mice, demonstrating a pro-inflammatory role of Arg-II in macrophages in aging.

Adipose tissue is composed of adipocytes and stromal vascular fraction cells. The latter is the major source for pro-inflammatory mediators produced from inflamed adipose tissues $[3,5]$. The dominant cells in the stromal vascular fraction are leukocytes and ATSCs. The former includes ATMs and lymphocytes, whereas the latter includes mainly pre-adipocytes and fibroblasts. It has been shown that ATMs are responsible for almost all adipose tissue TNF- $\alpha$ production and that ATSCs are the primary source for adipose tissue IL-6 [5, 8]. These cytokines have been shown to induce endothelial activation, and are associated with and predict atherosclerotic plaque progression $[43,44]$, suggesting a link between adipose tissue inflammation and age-associated diseases such as cardiovascular diseases and metabolic diseases.

It is to note that IL-6 was found in both ATMs and ATSCs in aging, suggesting that both cell types in aged adipose tissues contribute to age-associated adipose tissue inflammation. Interestingly, Arg-I expression was also found in both ATMs and ATSCs in aging, particularly when the Arg-II gene was ablated. Activated p38mapk was also found in ATMs and ATSCs of old WT mice, which was significantly reduced in the old $\mathrm{Arg}-\mathrm{II}^{-/-}$mice. Since the total p38mapk is enhanced in aging and reduced in $\mathrm{Arg}-\mathrm{II}^{-/-}$, and the ratio of p-p38/p38 is not significantly different between old WT and old Arg-II ${ }^{-/-}$mice adipose tissues, these results suggest the possibility that ArgII regulates total p38mapk levels in aging adipose tissues. This is different from the endothelial cells in which activation of p38mapk signaling is able to stimulate Arg-II ex- pression $[45,46]$. $\mathrm{p} 38$ mapk has been demonstrated to play critical roles in a wide variety of cellular processes, including adipose tissue function and inflammation [30, 31]. Adipose tissue-specific inhibition of p38mapk is effective in anti-obesity in mouse models [32]. Our present study suggests that $\mathrm{p} 38 \mathrm{mapk}$ inhibition could be also a target for inhibition of age-associated inflammation. P38mapk is a stress-responsive signaling that is involved in organismal aging and cellular senescence [47]. Previous studies show that overactive $\mathrm{p} 38$ mapk signaling plays an important role in endothelial dysfunction in aging and obesity $[46,48]$. Our present study extended this finding in the visceral adipose tissue in aging, demonstrating that total p38mapk is elevated in this tissue and involved in age-associated adipose tissue dysfunction, which is reduced by Arg-II deficiency. Since inhibition of p38mapk reduces IL- 6 release from aging adipose tissues as demonstrated by the present study, a role of p38mapk in Arg-II-promoted IL-6 production in aged adipose tissues could be proposed. The relevance of this finding is supported by the study of Starr et al. [6], showing that aged visceral adipose tissue produces more IL- 6 than that from young animals. Moreover, the relevance of our finding is further strengthened by clinical studies demonstrating that an elevated plasma IL-6 concentration in the elderly people predicts the 10year all-cause mortality [49]. It is presumable that adipose tissue inflammation cytokines could be released into circulation and contribute significantly to the chronic lowgrade systemic inflammation associated with aging. It remains interesting to investigate to what extent that adipose tissue-derived IL-6 contributes to the systemic inflammation, such as the plasma level of IL-6 in aging population. If this is confirmed, adipose tissues will present an important target organ for inflammaging and ageassociated chronic diseases.

In conclusion, the results of our study demonstrate that Arg-II plays an important role in the production of IL-6 from both ATMs and ATSCs of aged adipose tissues through p38mapk signaling. The study explores a novel function of Arg-II in adipose tissue inflammation, which may contribute to aging-associated diseases. Thus, targeting Arg-II and/or p38mapk might be beneficial in the treatment of age-associated inflammatory diseases.

\section{Statement of Ethics}

Animal work was approved by the Ethical Committee of Veterinary Office of Fribourg, Switzerland (No. 2011_12_FR, 21096), and was performed in compliance with guidelines on animal experimentation at our institution. 


\section{Disclosure Statement}

The authors declare no conflict of interest.

\section{Funding Sources}

This work was supported by the Swiss National Science Foundation (310030_141070/1 and 31003A_159582/1), Swiss Heart Foundation, and National Centres of Competence in Research Program (NCCR-Kidney.CH).

\section{Author Contributions}

J. Huang and C. Liu performed experiments, analyzed data, made graphics, and wrote the manuscript; X.-F. Ming and Z. Yang designed research, interpreted data, wrote the manuscript, and received funding for the research project.

\section{References}

1 Reddy KS. Global burden of disease study 2015 provides GPS for global health 2030. Lancet. 2016;388(10053):1448-9.

2 Costantino S, Paneni F, Cosentino F. Ageing, metabolism and cardiovascular disease. J Physiol. 2016;594(8):2061-73.

3 Weisberg SP, McCann D, Desai M, Rosenbaum M, Leibel RL, Ferrante AW Jr. Obesity is associated with macrophage accumulation in adipose tissue. J Clin Invest. 2003;112(12): 1796-808.

4 Lumeng CN, Bodzin JL, Saltiel AR. Obesity induces a phenotypic switch in adipose tissue macrophage polarization. J Clin Invest. 2007; 117(1):175-84

5 Lumeng CN, Liu J, Geletka L, Delaney C, Delproposto J, Desai A, et al. Aging is associated with an increase in $\mathrm{T}$ cells and inflammatory macrophages in visceral adipose tissue. J Immunol. 2011;187(12):6208-16.

6 Starr ME, Saito M, Evers BM, Saito H. Ageassociated increase in cytokine production during systemic inflammation-II: the role of IL- $1 \beta$ in age-dependent IL- 6 upregulation in adipose tissue. J Gerontol A Biol Sci Med Sci. 2015;70(12):1508-15.

7 Starr ME, Evers BM, Saito H. Age-associated increase in cytokine production during systemic inflammation: adipose tissue as a major source of IL-6. J Gerontol A Biol Sci Med Sci. 2009;64(7):723-30.

8 Ghosh AK, Mau T, O’Brien M, Garg S, Yung R. Impaired autophagy activity is linked to elevated ER-stress and inflammation in aging adipose tissue. Aging. 2016;8(10):2525-37.

9 Wu D, Ren Z, Pae M, Guo W, Cui X, Merrill $\mathrm{AH}$, et al. Aging up-regulates expression of inflammatory mediators in mouse adipose tissue. J Immunol. 2007;179(7):4829-39.

10 Harkins JM, Moustaid-Moussa N, Chung YJ, Penner KM, Pestka JJ, North CM, et al. Expression of interleukin- 6 is greater in preadipocytes than in adipocytes of 3T3-L1 cells and C57BL/6J and ob/ob mice. J Nutr. 2004; 134(10):2673-7.

11 Trim W, Turner JE, Thompson D. Parallels in immunometabolic adipose tissue dysfunction with ageing and obesity. Front Immunol. 2018;9:169.

12 Yepuri G, Velagapudi S, Xiong YY, Rajapakse AG, Montani JP, Ming XF, et al. Positive crosstalk between arginase-II and S6K1 in vascular endothelial inflammation and aging. Aging Cell. 2012;11:1005-16.

13 Xiong Y, Fru MF, Yu Y, Montani JP, Ming XF, Yang $\mathrm{Z}$. Long term exposure to $\mathrm{L}$-arginine accelerates endothelial cell senescence through arginase-II and S6K1 signaling. Aging. 2014; 6(5):369-79.

14 Shin WS, Berkowitz DE, Ryoo SW. Increased arginase II activity contributes to endothelial dysfunction through endothelial nitric oxide synthase uncoupling in aged mice. Exp Mol Med. 2012;44(10):594-602.

15 Khan M, Steppan J, Schuleri KH, Schuleri K, Ryoo S, Tuday E, et al. Upregulation of arginase-II contributes to decreased age-related myocardial contractile reserve. Eur J Appl Physiol. 2012;112(8):2933-41.

16 Kim JH, Bugaj LJ, Oh YJ, Bivalacqua TJ, Ryoo S, Soucy KG, et al. Arginase inhibition restores NOS coupling and reverses endothelial dysfunction and vascular stiffness in old rats. J Appl Physiol. 2009;107(4):1249-57.

17 Jenkinson CP, Grody WW, Cederbaum SD. Comparative properties of arginases. Comp Biochem Physiol B, Biochem Mol Biol. 1996; 114(1):107-32.

18 Ming XF, Rajapakse AG, Yepuri G, Xiong Y, Carvas JM, Ruffieux J, et al. Arginase II promotes macrophage inflammatory responses through mitochondrial reactive oxygen species, contributing to insulin resistance and atherogenesis. J Am Heart Assoc. 2012;1(4): e000992.

19 Masi S, Colucci R, Duranti E, Nannipieri M, Anselmino M, Ippolito C, et al. Aging modulates the influence of arginase on endothelial dysfunction in obesity. Arterioscler Thromb Vasc Biol. 2018;38(10):2474-83.

20 Yao L, Bhatta A, Xu Z, Chen J, Toque HA, Chen Y, et al. Obesity-induced vascular inflammation involves elevated arginase activity. Am J Physiol Regul Integr Comp Physiol. 2017;313(5):R560-71.

21 Yang Z, Ming XF. Arginase: the emerging therapeutic target for vascular oxidative stress and inflammation. Front Immunol. 2013;4: 149.

22 Liu C, Rajapakse AG, Riedo E, Fellay B, Bernhard MC, Montani JP, et al. Targeting arginase-II protects mice from high-fat-diet-in- duced hepatic steatosis through suppression of macrophage inflammation. Sci Rep. 2016; 6:20405.

$23 \mathrm{Hu} \mathrm{H}$, Moon J, Chung JH, Kim OY, Yu R, Shin MJ. Arginase inhibition ameliorates adipose tissue inflammation in mice with dietinduced obesity. Biochem Biophys Res Commun. 2015;464(3):840-7.

24 Atawia RT, Toque HA, Meghil MM, Benson TW, Yiew NKH, Cutler CW, et al. Role of arginase 2 in systemic metabolic activity and adipose tissue fatty acid metabolism in dietinduced obese mice. Int J Mol Sci. 2019;20(6): E1462.

25 Navarro LA, Wree A, Povero D, Berk MP, Eguchi A, Ghosh S, et al. Arginase 2 deficiency results in spontaneous steatohepatitis: a novel link between innate immune activation and hepatic de novo lipogenesis. J Hepatol. 2015;62(2):412-20.

26 Kim SY, Lim EJ, Yoon YS, Ahn YH, Park EM, Kim HS, et al. Liver X receptor and STAT1 cooperate downstream of Gas6/Mer to induce anti-inflammatory arginase 2 expression in macrophages. Sci Rep. 2016;6:29673.

27 Hardbower DM, Asim M, Murray-Stewart T, Casero RA Jr, Verriere T, Lewis ND, et al. Arginase 2 deletion leads to enhanced M1 macrophage activation and upregulated polyamine metabolism in response to helicobacter pylori infection. Amino Acids. 2016;48(10): 2375-88.

28 Cuadrado A, Nebreda AR. Mechanisms and functions of p38 MAPK signalling. Biochem J. 2010;429(3):403-17.

29 Kumar S, Boehm J, Lee JC. p38 MAP kinases: key signalling molecules as therapeutic targets for inflammatory diseases. Nat Rev Drug Discov. 2003;2(9):717-26.

30 Tomida T, Takekawa M, Saito H. Oscillation of p38 activity controls efficient pro-inflammatory gene expression. Nat Commun. 2015; 6:8350.

31 Westermann D, Rutschow S, Van Linthout S, Linderer A, Bücker-Gärtner C, Sobirey M, et al. Inhibition of p38 mitogen-activated protein kinase attenuates left ventricular dysfunction by mediating pro-inflammatory cardiac cytokine levels in a mouse model of diabetes mellitus. Diabetologia. 2006;49(10): 2507-13. 
32 Zhang S, Cao H, Li Y, Jing Y, Liu S, Ye C, et al. Metabolic benefits of inhibition of p38a in white adipose tissue in obesity. PLoS Biol. 2018;16(5):e2004225.

33 Mancuso P, Bouchard B. The impact of aging on adipose function and adipokine synthesis. Front Endocrinol. 2019;10:137.

34 Shi O, Morris SM Jr, Zoghbi H, Porter CW, O'Brien WE. Generation of a mouse model for arginase II deficiency by targeted disruption of the arginase II gene. Mol Cell Biol. 2001;21:811-3.

35 Xiong Y, Yepuri G, Montani JP, Ming XF, Yang Z. Arginase-II deficiency extends lifespan in mice. Front Physiol. 2017;8:682.

36 Xiong Y, Yepuri G, Necetin S, Montani JP, Ming XF, Yang Z. Arginase-II promotes tumor necrosis factor- $\alpha$ release from pancreatic acinar cells causing $\beta$-cell apoptosis in aging. Diabetes. 2017;66(6):1636-49.

37 Mau T, Yung R. Adipose tissue inflammation in aging. Exp Gerontol. 2018;105:27-31.

38 Ni Y, Ni L, Zhuge F, Xu L, Fu Z, Ota T. Adipose tissue macrophage phenotypes and characteristics: the key to insulin resistance in obesity and metabolic disorders. Obesity. 2020; 28(2):225-34.
39 Ricardo SD, van Goor H, Eddy AA. Macrophage diversity in renal injury and repair. J Clin Invest. 2008;118(11):3522-30.

40 Munder M, Eichmann K, Morán JM, Centeno F, Soler G, Modolell M. Th1/Th2-regulated expression of arginase isoforms in murine macrophages and dendritic cells. J Immunol. 1999;163(7):3771-7.

41 Khallou-Laschet J, Varthaman A, Fornasa G, Compain C, Gaston AT, Clement M, et al. Macrophage plasticity in experimental atherosclerosis. PloS One. 2010;5(1):e8852.

42 Wang XP, Zhang W, Liu XQ, Wang WK, Yan F, Dong WQ, et al. Arginase I enhances atherosclerotic plaque stabilization by inhibiting inflammation and promoting smooth muscle cell proliferation. Eur Heart J. 2014;35(14): 911-9.

43 Zhang J, Alcaide P, Liu L, Sun J, He A, Luscinskas FW, et al. Regulation of endothelial cell adhesion molecule expression by mast cells, macrophages, and neutrophils. PLoS One. 2011;6(1):e14525.

44 Eltoft A, Arntzen KA, Wilsgaard T, Mathiesen EB, Johnsen SH. Interleukin-6 is an independent predictor of progressive atherosclerosis in the carotid artery: the Tromso Study. Atherosclerosis. 2018;271:1-8.
45 Shatanawi A, Romero MJ, Iddings JA, Chandra S, Umapathy NS, Verin AD, et al. Angiotensin II-induced vascular endothelial dysfunction through RhoA/Rho kinase/p38 mitogen-activated protein kinase/arginase pathway. Am J Physiol Cell Physiol. 2011; 300(5):C1181-92.

46 Wu Z, Yu Y, Liu C, Xiong Y, Montani JP, Yang Z, et al. Role of p38 mitogen-activated protein kinase in vascular endothelial aging: interaction with arginase-II and S6K1 signaling pathway. Aging. 2015;7(1):70-81.

47 Freund A, Patil CK, Campisi J. p38MAPK is a novel DNA damage response-independent regulator of the senescence-associated secretory phenotype. EMBO J. 2011;30(8):153648.

48 Yu Y, Rajapakse AG, Montani JP, Yang Z, Ming XF. p38 mitogen-activated protein kinase is involved in arginase-II-mediated eNOS-Uncoupling in obesity. Cardiovasc Diabetol. 2014;13:113

49 Varadhan R, Yao W, Matteini A, Beamer BA, Xue QL, Yang H, et al. Simple biologically informed inflammatory index of two serum cytokines predicts 10 year all-cause mortality in older adults. J Gerontol A Biol Sci Med Sci. 2014;69(2):165-73. 